\title{
De la fonction comparateur à la centrale d'alarme
}

\author{
Patrick LAGONOTTE ${ }^{1}$, Dominique $\mathrm{JACOB}^{2}$ \\ I.U.T. de Poitiers, 6 allée Jean MONNET \\ 86010 Poitiers Cedex \\ ${ }^{1}$ Département Hygiène, Sécurité, Environnement, site de Niort lagonotte@let.ensma.fr \\ ${ }^{2}$ Département Génie Electrique et Informatique Industrielle, site de Poitiers dominique.jacob@univ-poitiers.fr
}

RÉSUMÉ : L’enseignement de l'électricité dans les départements Hygiène Sécurité Environnement (HSE) des I.U.T. ne comprend qu'un faible nombre d'heures en première et en deuxième année. Il est cependant intéressant d'utiliser ce peu de temps à la présentation d'applications de l'électricité liées à la sécurité. Dans ce cadre, ce T.P. associe la découverte de l'électronique et traite des systèmes d'alarme. En utilisant uniquement les notions de base : pont diviseur de tension, comparateur de tensions, fonction logique (porte NAND), il est possible de mettre en œuvre et d'étudier le fonctionnement d'un système d'alarme incendie pourvu d'un système d'autosurveillance.

Mots clés : Fonctions électroniques, comparateur, alarme incendie, analyse système.

\section{INTRODUCTION}

Le public visé est celui des étudiants de première année du D.U.T. Hygiène Sécurité Environnement. L’objectif de l'enseignement d'électricité dans cette discipline n'est pas de former des électriciens, mais d'inculquer des notions de base relatives au fonctionnement des systèmes électriques liés à la sécurité.

Des alarmes se rencontrent sur un grand nombre d'objets technologiques. Dans tous les cas une fonction comparateur permet de comparer le niveau d'une grandeur physique à celui d'un seuil à ne pas dépasser.

Donnons comme exemples concrets : le voyant rouge de surchauffe du moteur thermique d'une automobile qui prévient le conducteur d'un problème ou d'un fonctionnement non correct ; le voyant jaune du niveau bas de la jauge à carburant qui prévient le conducteur d'un possible problème à venir.

La séance de travaux pratiques comprend l'étude de différents montages comparateurs à amplificateurs opérationnels. Cependant, l'assemblage de plusieurs fonctions élémentaires comme des comparateurs permet d'obtenir un système d'alarme simple avec une auto surveillance du montage.

L'analyse d'un système électronique (ou même mécanique) n'est pas simple à ce niveau d'étude. Il apparaît un manque d'habitude ou de culture des étudiants pour appréhender et raisonner sur une apparente complexité due à l'assemblage et à l'enchaînement de différentes fonctions.

L'objectif de ce document est de présenter les montages de travaux pratiques qui ont été élaborés à partir d'amplificateurs opérationnels allant jusqu'au montage d'un comparateur à fenêtre associé à des boîtiers déclencheurs manuels (DM) pour réaliser une petite alarme incendie.

\section{LE MONTAGE COMPARATEUR}

\subsection{Description du montage}

Dans une premier temps les étudiants vont se familiariser avec la carte électronique comportant le montage comparateur. La carte électronique présentée figure 1 comporte :

- trois bornes d'alimentation (+12 V, masse, $-12 \mathrm{~V})$;

- deux potentiomètres linéaires permettant de faire varier les tensions des points milieux entre en \pm 12 $\mathrm{V}$;

- un jeux de quatre bornes permettant d'inverser les entrées e+ et e- du comparateur ;

- un comparateur réalisé à l'aide d'un amplificateur opérationnel en boucle ouverte ;

- deux LED en sortie (une rouge et une verte) permettant de visualiser l'état de la sortie du comparateur.

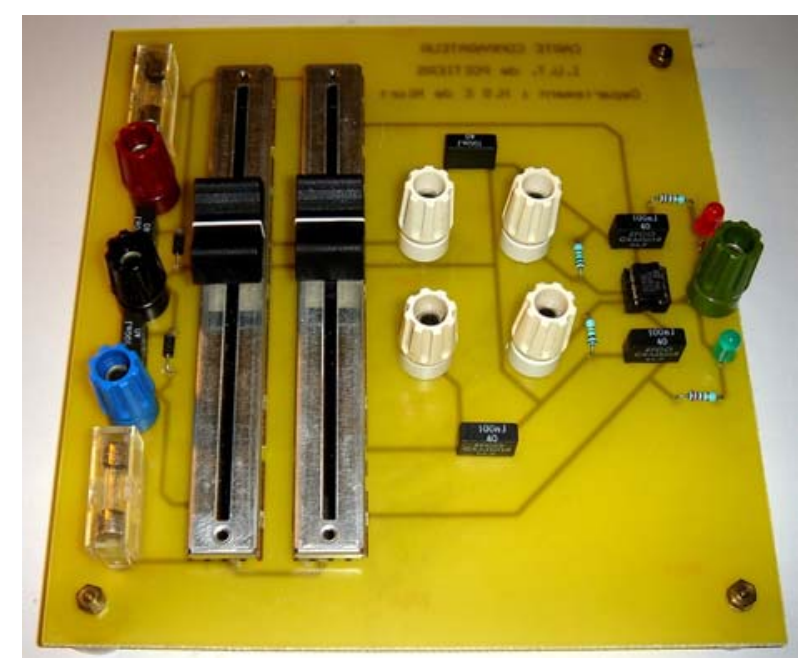

Fig. 1 : Carte électronique comparateur 
Le montage électronique correspondant est présenté figure 3.

Les alimentations sont protégées par des diodes et des fusibles en cas d'inversion des polarités, de manière à protéger l'amplificateur opérationnel d'une éventuelle destruction (Figure 2). Des condensateurs sont utilisés pour effectuer le découplage des alimentations de l’amplificateur opérationnel.

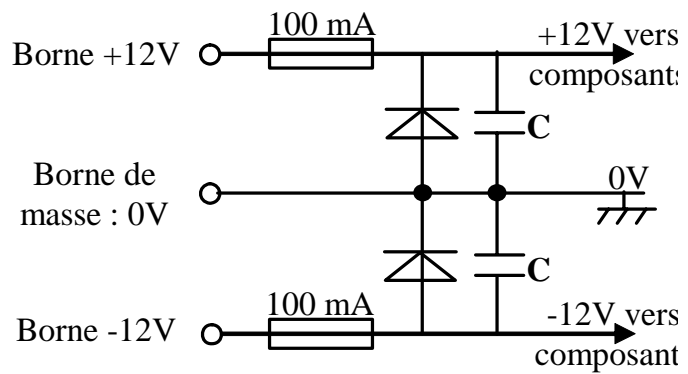

Fig. 2 : Protection du montage en cas d'inversion des polarités de l'alimentation

\subsection{Fonctionnement manuel}

Dans un premier temps les étudiants utilisent le montage en mode manuel de manière à analyser le fonctionnement du comparateur (figure 3).

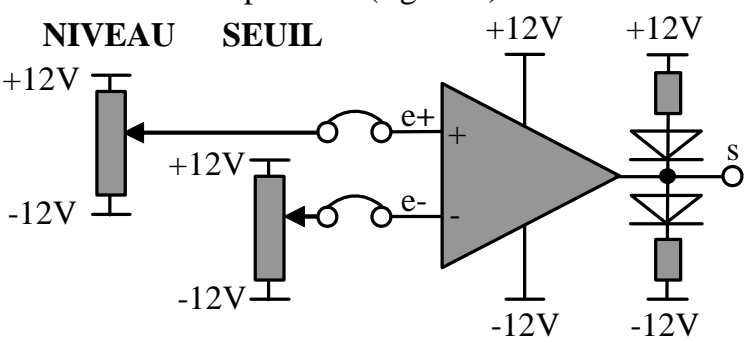

Fig. 3 : Schéma électronique de la carte comparateur

Il est possible de brancher un voltmètre continu au niveau des entrées e+ et e- du comparateur pour vérifier qu'il y a bien concordance entre les positions des potentiomètres, la différence de tension entre les entrées, et la LED qui s'éclaire en sortie.

En inversant des branchements au niveau des entrées e+ et e- les étudiants vérifient que l'on passe bien d'une alarme par dépassement positif à une alarme par dépassement négatif. Pour nos étudiants, nous pouvons alors parler du cas concret d'une rivière en introduisant les notions d'alarme par dépassement positif pour un niveau de crue et d'alarme par dépassement négatif pour un niveau d'étiage. Nous verrons plus loin que cette analogie hydraulique pourra resservir au niveau de l'alarme incendie.

\subsection{Fonctionnement autonome avec un GBF}

L'objectif est de montrer que les comparateurs peuvent fonctionner de manière très rapide devant les constantes de temps humaines.

Pour cela nous allons remplacer l'entrée niveau par le signal délivré par un GBF, et observer les différents signaux à l'aide d'un oscilloscope (figure 4). Le signal du GBF étant appliqué directement sur l'entrée e+ du comparateur.

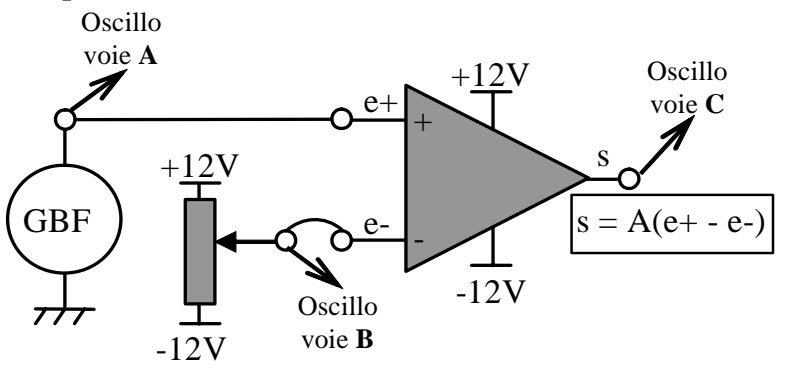

Fig. 4 : Montage comparateur utilisé avec un GBF

Dans ce simple cas où il est nécessaire d’observer trois voies différentes de manière simultanée. Une difficulté rencontrée par les étudiants est l'analyse et l'interprétation des trois signaux sur un oscilloscope analogique à deux voies. L'utilisation d'un oscilloscope numérique à quatre voies avec écran couleur peut être un plus pour une meilleure compréhension et analyse des différentes courbes.

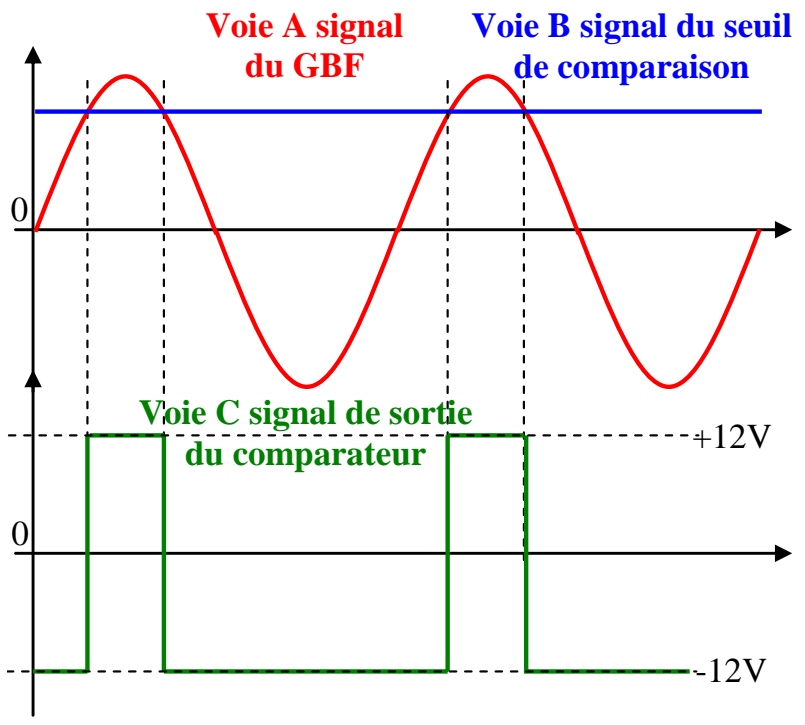

Fig. 5 : Exemple de courbes visualisées

La fréquence du signal utilisée est de l'ordre de $1 \mathrm{kHz}$ à $10 \mathrm{kHz}$ : suffisamment grande pour avoir une trace stable à l'oscilloscope, et suffisamment faible pour ne pas faire apparaître les imperfections des amplificateurs opérationnels.

A ce niveau du TP, il est important de sensibiliser les étudiants au cas de comparateurs devant répondre très rapidement. C'est le cas d'un système " airbag » de sécurité dans les automobiles. Le comparateur traitant le signal issu d'un accéléromètre pour déclencher les moyens pyrotechniques de gonflage rapide des coussins de sécurité. 


\section{LE MONTAGE ALARME INCENDIE}

\subsection{Présentation du montage}

L'objectif est maintenant de passer à l'étude d'un système comportant plusieurs comparateurs (deux dans notre cas) et une porte NAND.

Le montage utilisé (voir figure 6) comporte :

- une carte électronique alimentée en monotension 012 V (à gauche) ;

- trois déclencheurs manuels d'alarme incendie (DM), équipés pour notre montage de bornes de 4 $\mathrm{mm}$;

- une résistance de fin de boucle (à droite), montée avec des bornes de $4 \mathrm{~mm}$.

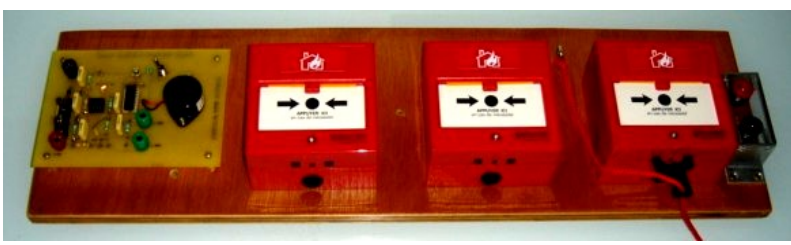

Fig. 6 : La maquette alarme incendie

La carte électronique comporte uniquement deux circuits intégrés: un double amplificateur opérationnel TL082 et une quadruple porte NAND en technologie CMOS 4011 dont une seule porte est utilisée.

\subsection{Protection de l'alimentation}

L'alimentation est protégée par une diode et un fusible en cas d'inversion des polarités, de manière à protéger les circuits intégrés d'une éventuelle destruction (figure 7). Des condensateurs sont utilisés pour effectuer le découplage de l'alimentation des deux circuits.

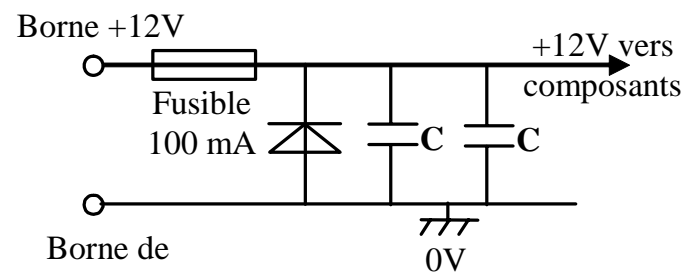

masse : $0 \mathrm{~V}$

Fig. 7 : Protection du montage en cas d'inversion des polarités de l'alimentation

\subsection{Analyse du fonctionnement du premier pont di- viseur de tension}

L'analyse du montage par les étudiants comprend l'étude de deux ponts diviseurs de tension.

Le premier pont (figure 8) est constitué par trois résistances identiques et permet de générer des tensions de référence de $\mathrm{V} 1=4 \mathrm{~V}$ et $\mathrm{V} 2=8 \mathrm{~V}$. Ces tensions sont stabilisées par des condensateurs et servent de références aux entrées des comparateurs de tension. Ce montage correspond à un comparateur à fenêtre.

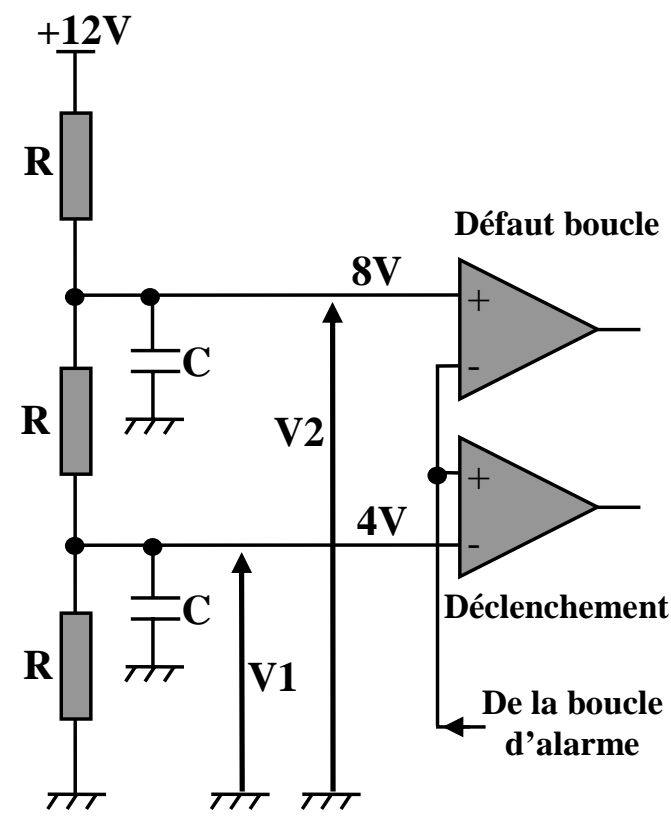

Fig. 8 : Premier pont diviseur à trois résistances

\subsection{Analyse du fonctionnement du second pont di-} viseur de tension

Le second pont diviseur (figure 9) constitue la boucle de déclenchement de l'alarme avec les déclencheurs manuels branchés en parallèle sur la résistance de fin de boucle.

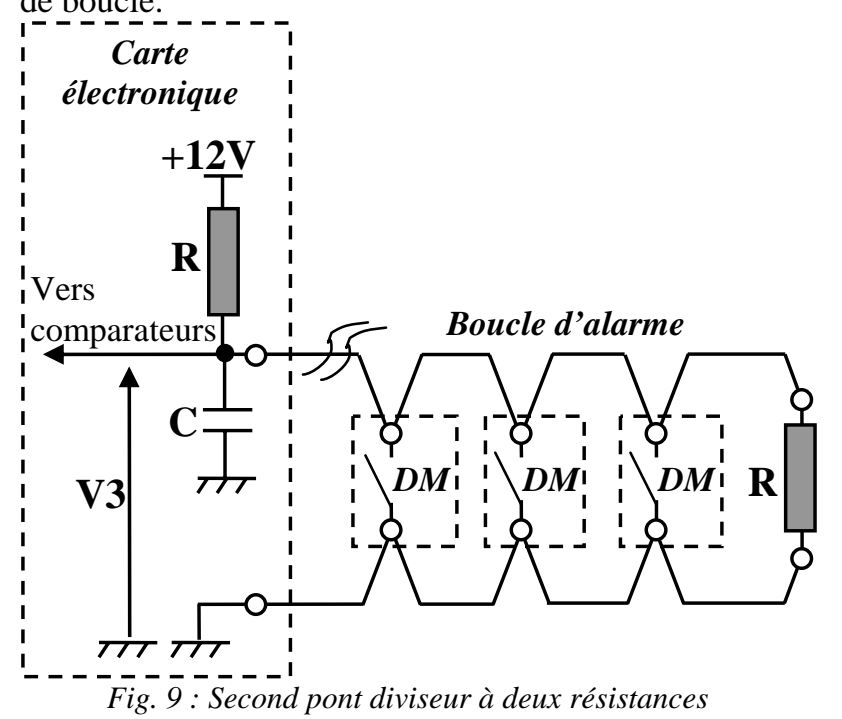

Ce pont est constitué par deux résistances de valeur identiques, cependant les éléments extérieurs permettent d'avoir trois cas de fonctionnement :

- dans le cas «normal», le pont diviseur de tension permet d'obtenir une tension $\mathrm{V} 3=6 \mathrm{~V}$;

- en cas d'action sur un des déclencheurs manuels, un contact se ferme et la tension $\mathrm{V} 3$ passe à $0 \mathrm{~V}$;

- en cas de débranchement d'un fil au niveau de la boucle, la résistance de fin de boucle n'est plus alimentée et la tension V3 passe à 12V. 
Les trois cas de fonctionnement peuvent être facilement vérifiés avec les étudiants en plaçant un voltmètre continu au niveau des deux bornes de départ de la boucle d'alarme sur la carte électronique.

Le courant circulant en permanence en fonctionnement normal dans la boucle d'alarme jusqu'à la résistance de fin de boucle permet en fait une auto-surveillance permanente du montage. C'est ce principe simple qui est utilisé sur tous les systèmes d'alarme filaire (intrusion ou incendie).

\section{Remarque :}

Dans le cas de ce montage :

- la résistance de fin de boucle est apparente, en pratique la résistance de fin de boucle est disposée à l'intérieur du dernier boitier.

- le câblage est effectué avec de classiques fils de TP pourvus de fiches de $4 \mathrm{~mm}$. Dans la pratique un câble à deux conducteurs part de la centrale d'alarme et relie les différents boitiers de déclenchement manuel.

\subsection{Analyse du fonctionnement des comparateurs}

Regardons maintenant le rôle des comparateurs de tension (figure 10). Si la circulation du courant dans la boucle est interrompue, la tension V3 de la boucle d'alarme passe au-dessus de 8V. La tension en sortie du comparateur haut passe alors à $0 \mathrm{~V}$, et la LED d’indication de défaut de boucle s’éclaire.

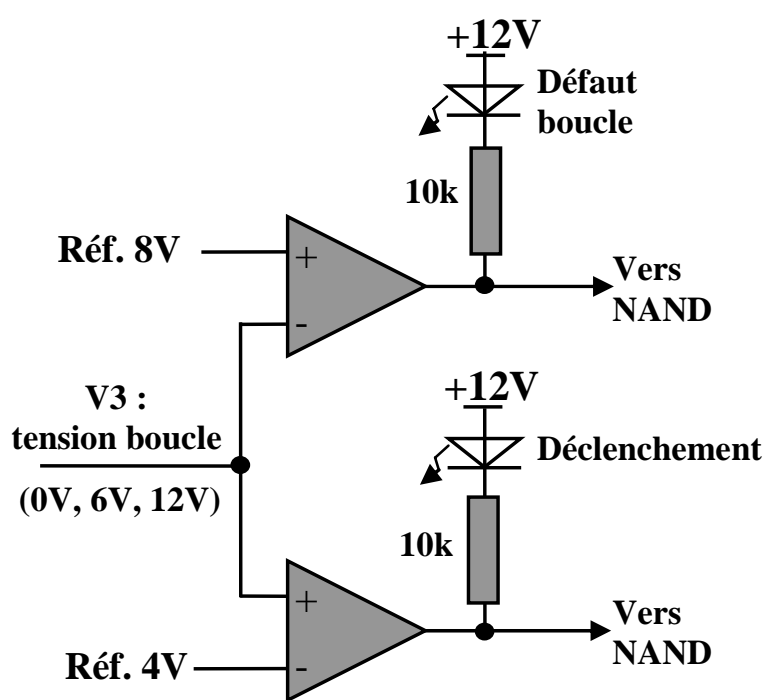

Fig. 10 : Comparateurs et signalisation des états

Si un déclencheur manuel est actionné, la tension V3 de la boucle d'alarme passe en dessous de $4 \mathrm{~V}$. La tension en sortie du comparateur bas passe alors à $0 \mathrm{~V}$, et la LED d'indication de déclenchement de l'alarme s'éclaire.

Finalement en reprenant l'analogie de la rivière, nous avons une détection de crue $(\mathrm{V} 3>8 \mathrm{~V})$, une détection d'étiage (V3 $<4 \mathrm{~V})$, et un niveau normal $(4 \mathrm{~V}<\mathrm{V} 3<$ $8 \mathrm{~V})$.

\subsection{Traitement des signaux d'alarmes}

Nous disposons en sortie de chaque comparateur d'une information logique $(0 \mathrm{~V}-12 \mathrm{~V})$ qui peut être traitée directement par un circuit logique en technologie CMOS. Nous souhaitons qu'en cas de défaut de la boucle ou de déclenchement manuel qu'une alarme sonore soit activée. Pour cela nous allons utiliser une porte NAND comme présentée figure 11.

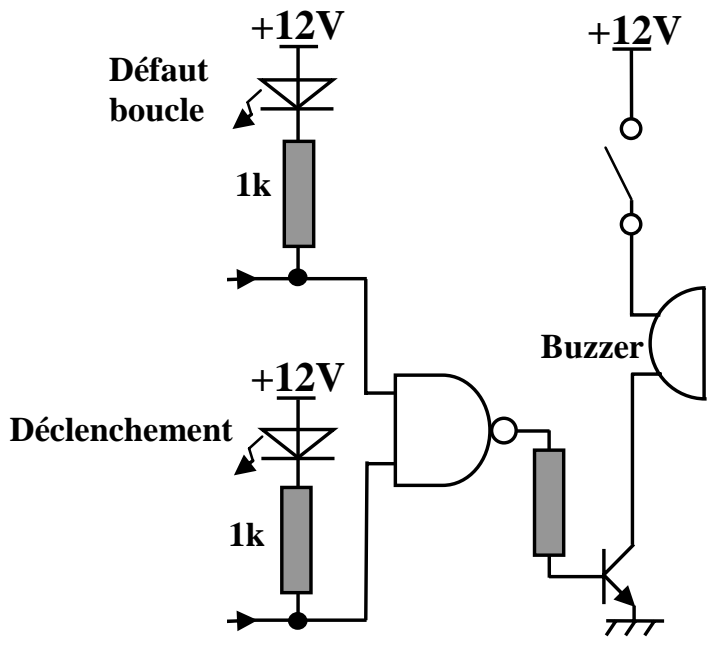

Fig. 11 : Traitement de la signalisation

Ces deux informations logiques permettent d'obtenir quatre combinaisons possibles. Cependant la tension analogique V3 ne peut pas être simultanément supérieure à $8 \mathrm{~V}$ et inférieure à $4 \mathrm{~V}$, ce qui élimine une solution et n'en laisse plus que trois (nous retrouvons les trois niveaux analogiques).

Il paraît important à ce niveau de synthétiser le fonctionnement de cette partie du montage sous la forme d'un tableau.

\begin{tabular}{|c|c|c|c|c|c|c|}
\hline \multirow{3}{*}{$\begin{array}{c}\text { Tension } \\
\text { Boucle V3 }\end{array}$} & & & \multicolumn{3}{|c|}{ Porte NAND } & \multirow{3}{*}{ Buzzer } \\
\hline & \multicolumn{2}{|c|}{ LED } & & & $\begin{array}{l}\text { Sor- } \\
\text { tie }\end{array}$ & \\
\hline & $\begin{array}{l}\text { Dé- } \\
\text { faut }\end{array}$ & $\begin{array}{l}\text { Dé- } \\
\text { cl. }\end{array}$ & $\mathrm{A}$ & B & $S$ & \\
\hline $\begin{array}{c}\mathrm{V3}<4 \mathrm{~V} \text { et } \\
8 \mathrm{~V}<\mathrm{V} 3\end{array}$ & Imp. & Imp. & 0 & 0 & 1 & Imp. \\
\hline $8 \mathrm{~V}<\mathrm{V3}$ & 1 & 0 & 0 & 1 & 1 & 1 \\
\hline $\mathrm{V} 3<4 \mathrm{~V}$ & 0 & 1 & 1 & 0 & 1 & 1 \\
\hline $4 \mathrm{~V}<\mathrm{V} 3<8 \mathrm{~V}$ & 0 & 0 & 1 & 1 & 0 & 0 \\
\hline
\end{tabular}

Tab. 1 : Traitement de la signalisation par la porte NAND

La sortie de la porte NAND commande un buzzer par l'intermédiaire d'un transistor permettant d'amplifier le courant. Un interrupteur permet de neutraliser le buzzer dont le niveau sonore n'est pas négligeable (80 $\mathrm{dB})$. 
Ce système est purement analogique. Cependant un système de traitement d'alarme numérique programmable peut facilement être associé à ce montage, pour ajouter des fonctions comme une télétransmission des informations, la date, l'heure et le numéro de la boucle de déclenchement ou du disfonctionnement.

\section{Remarque :}

Les systèmes d'alarme incendie industriels sont à 4 niveaux analogiques permettant de détecter :

- un circuit ouvert ;

- l'état normal (fermeture sur une résistance de fin de boucle);

- un déclenchement d'un déclencheur manuel (fermeture sur une résistance de DM) ;

- un court-circuit.

Le traitement des disfonctionnements (circuit ouvert et court-circuit) pouvant être traités par le signalement d'un impératif besoin de maintenance.

D'autre part les systèmes d'alarme demandent une alimentation autonome sur batterie de manière à assurer leur fonction même en cas de coupure d'électricité du secteur.

\subsection{Cas des alarmes d'intrusion filaires}

Dans le cas d'une alarme intrusion les capteurs sont des contacts normalement fermés disposés en série alors que pour une centrale d'alarme incendie ce sont des contacts normalement ouverts disposés en parallèle.

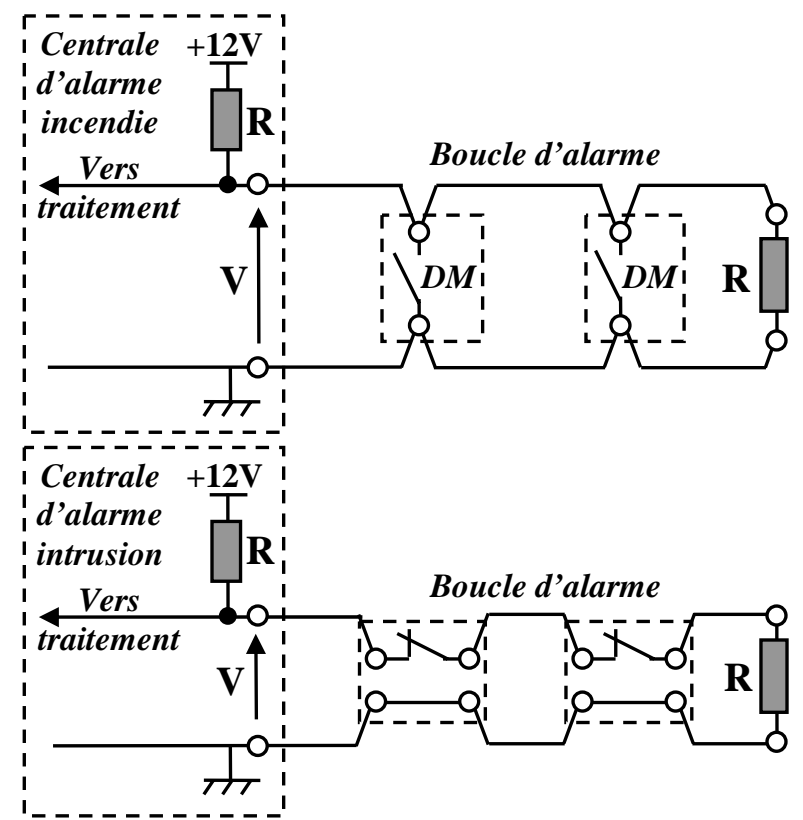

Fig. 12 : Les différentes structures de boucles d'alarme

Les systèmes d'alarme intrusion sont plus compliqués par le fait qu'ils disposent en général d'une seconde boucle dite «boucle de protection ». Le rôle de cette boucle de protection est de déclencher l'alarme en cas de tentative d'accès au câblage par ouverture des diffé- rents éléments pour tentative de neutralisation par exemple. Les câbles utilisés dans le cas d'une alarme intrusion sont constitués de deux paires blindées.

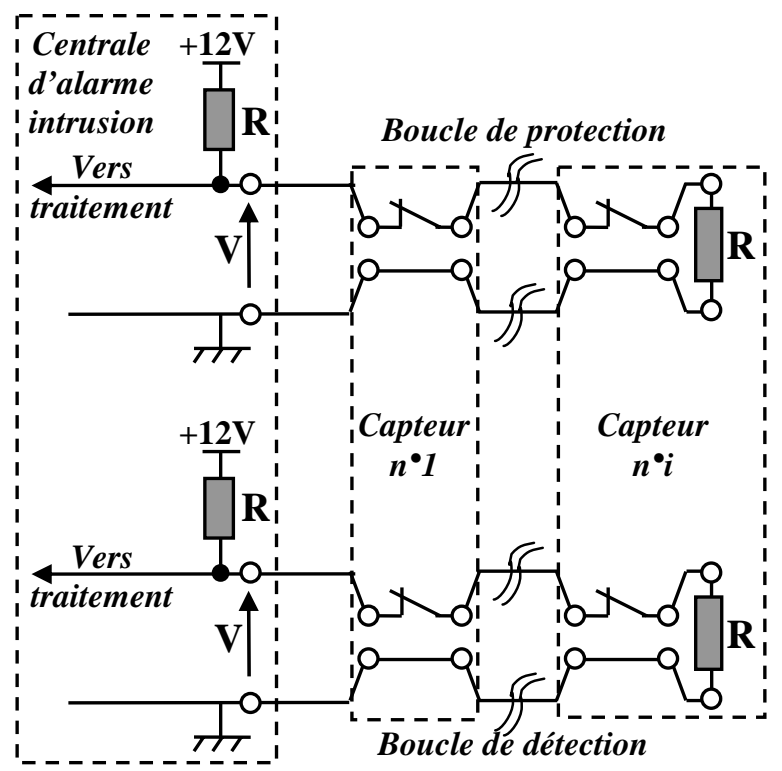

Fig. 13 : Structure à double boucle d'une alarme intrusion

\section{CONCLUSION}

Bien que l'étude du montage comparateur de tension (boucle ouverte) soit en général passée rapidement au profit de montages plus élaborés à amplificateurs opérationnels (boucle fermée), ils font l'objet d'applications industrielles.

L’étude de tels montages peut s’avérer intéressante au niveau des lycées dans le cadre de l'initiation à l'électricité et à l'électronique.

Ces différents montages à faibles coûts de revient et simples à réaliser permettent d'effectuer l'étude d'un comparateur pour aller vers l'étude d'un système électronique.

Une difficulté rencontrée par les étudiants est l'enchainement des fonctions. Cependant cette approche est indispensable pour effectuer une analyse système, et c'est cette méthode qui est utilisée pour construire des systèmes industriels.

Après 3 années d'utilisation, ce TP est jugé très intéressant par les étudiants, qui disposent des connaissances nécessaires à l'analyse du fonctionnement du montage. Cependant, la difficulté provient toujours de la nécessité d'assembler et de structurer leurs différentes connaissances pour arriver à l'analyse globale du système. Un intéressant challenge intellectuel à résoudre pour une majorité d'étudiants, malheureusement une difficulté parfois insurmontable pour d'autres. 
Ces systèmes industriels dont l'étude est parfois négligée peuvent devenir compliqués lorsque l'on a à faire avec des capteurs adressables par courants porteurs.

\section{Remerciement}

Nous tenons à remercier $\mathrm{M}$. Jean-Claude JOULAIN qui a participé à la réalisation de ces maquettes électroniques.

\section{REFERENCES}

Catalogue Merlin-Gérin, Sécurité des bätiments, 2004

Catalogue Legrand 2007, produits et systèmes pour installations électriques et réseaux d’information.

Documents techniques TALCO :

- Livret conseil (dossier technico-commercial alarme) ;

- Centrale filaire ACBX 24, notice d'installation et d'utilisation.

Document technique Cooper-Nugelec :

- Notice d'utilisation ECA 4000, 2, 4 et 8 boucles 256 points avec CMSI de type A 4 lignes ET-MT. 